\title{
SAR Image Registration in the Presence of Rotation and Translation: A Constrained Least Squares Approach
}

\author{
Luca Pallotta, Senior Member, IEEE, Gaetano Giunta, Senior Member, IEEE, and Carmine Clemente, Senior \\ Member, IEEE
}

\begin{abstract}
This letter proposes a coregistration algorithm to compensate for possible inaccuracy of trajectory sensor during the SAR image acquisition process. Such a misalignment can be modeled as a pure displacement in range and azimuth directions and a rotation effect due to different angle of sight. The approach is formalized as a Constrained Least Squares (CLS) optimization problem enforcing a constraint of absence of a zooming effect between the two SAR images. Moreover, system equations can optionally be weighted according to local properties between the extracted patches within the quoted couple. Interestingly, the solution can be obtained in closed-form, therefore with a low computational cost. The results of the tests conducted on the 9.6 GHz Gotcha SAR data demonstrate the capability of the strategy to proper register the imagery.
\end{abstract}

Index Terms-Synthetic Aperture Radar, SAR coregistration, Rotated and Translated Images.

\section{INTRODUCTION}

The growing interest in Synthetic Aperture Radar (SAR) applications for environmental monitoring, such as the fire detection, the study of the evolution of glacier melting and so on, increasingly requires the availability of perfectly coregistered SAR image series. Nonetheless, it is very difficult for the flying sensor to maintain the same trajectory during the acquisition campaign with a negligible error. This latter directly reflects on the set of images that will present a possible translation and rotation effects or a combination of them. To overcome this drawback, a possible solution is the coregistration, where one image is selected as master and all the remaining, referred to as slave, are re-aligned with respect to it to have that the corresponding pixels in the images refer to the same point within the scene [1]. Coregistration is typically performed by searching for the corresponding tie points between the master and slave images whose respective location inside them gives a measure of their misalignment. Once the tie points have been identified, the slave image is properly shifted in azimuth and range directions, and also resampled, in order to perfectly superimpose it to the master. Over the years, a pletora of works has been published in this context. Some of them are based on the exploitation of the 2-Dimensional (2D) cross-correlation between master and

L. Pallotta and G. Giunta are with the Department of Engineering, University of Roma Tre, via Vito Volterra 62, 00146 Rome, Italy (e-mail: luca.pallotta@uniroma3.it, gaetano.giunta@uniroma3.it).

C. Clemente is with the Department of Electronic and Electrical Engineering, University of Strathclyde, G1 1XW Glasgow, Scotland (e-mail: carmine.clemente@strath.ac.uk). slave to identify the quoted displacement [2], [3]. Similarly, other works exploit the properties of the Fourier domain to enhance the registration capabilities as well as the computation efficiency [4]-[6], moreover, geometrical approaches are also applied to this context [7]. Finally, some alternatives to the classic approaches can be found in [8], [9], where featurebased methods characterized by a low computational cost are also devised.

The above mentioned coregistration approaches are generally designed in a way such that the slave images are aligned to the master by means of a rigid translation in range and azimuth. In practice, however, the two or more images in question may be affected by other types of displacement effects due to the variations in sensor trajectory during the acquisition process. These phenomenologies may be synthesized in a pure displacement in range and azimuth directions and a rotation effect due to the different angle of sight. This letter proposes a method to formalize the problem of SAR images coregistration when not only there is a rigid shift between the two images but also a rotation. In addition, since the two SAR images have the same resolution and the same swath on terrain, i.e., they are not affected by a zooming effect, a constraint on the scaling factor between them is also enforced. Accordingly, the images are divided into several patches and the relative misalignment among them is estimated as the peak of the 2D cross-correlation; then, the resulting problem is formulated in terms of a Constrained Least Squares (CLS) approach, whose solution is efficiently found resorting to the theory of Generalized Trust Region Subproblems (GTRSs) [10]. The analyses are conducted on the challenging Gotcha SAR data acquired at $9.6 \mathrm{GHz}$ [11], showing the effectiveness and usefulness of the derived methodology. It is worth to recall that the devised problem follows the line of reasoning provided in [12], where the unconstrained version is formalized to estimate the spatial shift, change of scale, and rotation that account for a global motion component in video coding.

The letter is organized as follows. In Section II the problem formulation is given together with its solution; in addition, the overall coregistration algorithm is described in details. The conducted tests are reported in Section III to demonstrate the effectiveness of the proposed approach on the challenging Gotcha SAR images. Conclusions and hints for future works are confined to Section IV. 


\section{A. Notation}

We use boldface lower case for vectors $a$ and upper case for matrices $\boldsymbol{A}$. The $k$-th entry of $\boldsymbol{a}$ is denoted by $\boldsymbol{a}(k)$, and the $(k, n)$-th entry of $\boldsymbol{A}$ is denoted by $\boldsymbol{A}(k, n)$. Then, $\operatorname{diag}(\boldsymbol{a})$ is the diagonal matrix whose diagonal entries are the values in vector $\boldsymbol{a}$. As to numerical sets, $\mathbb{C}$ is the set of complex numbers, and $\mathbb{C}^{K \times N}$ is the Euclidean space of $(K \times N)$-dimensional complex matrices (or vectors if $N=1$ ), whereas $\mathbb{S}^{N}$ is the set of $N \times N$ symmetric matrices. The curled inequality symbol $\succeq$ is used to indicate generalized matrix inequality: for any $\boldsymbol{A} \in \mathbb{C}^{N}, \boldsymbol{A} \succeq \mathbf{0}$ means that $\boldsymbol{A}$ is a positive semi-definite matrix. $\lambda_{1}(\boldsymbol{X}), \ldots, \lambda_{N}(\boldsymbol{X})$, with $\lambda_{1}(\boldsymbol{X}) \geq \ldots \geq \lambda_{N}(\boldsymbol{X})$, denote the eigenvalues of $\boldsymbol{X} \in \mathbb{S}^{N}$, arranged in decreasing order. Furthermore, given $\boldsymbol{B} \succ \mathbf{0}$ and $\boldsymbol{A} \in \mathbb{S}^{N}$, the generalized eigenvalues of the matrix pair $(\boldsymbol{A}, \boldsymbol{B})$ are given by $\lambda_{i}(\boldsymbol{A}, \boldsymbol{B})=\lambda_{i}\left(\boldsymbol{B}^{-1 / 2} \boldsymbol{A} \boldsymbol{B}^{-1 / 2}\right)$, $i=1, \ldots, N$. The symbols $(\cdot)^{*},(\cdot)^{T}$, and $(\cdot)^{\dagger}$ denote the conjugate, transpose, and conjugate transpose operators, respectively, while $\|\cdot\|$ is the Euclidean matrix norm. Finally, $j=\sqrt{-1}$ is the imaginary unit.

\section{PROBlem Formulation AND COREgistration ALGORITHM}

This section describes the proposed algorithm for SAR images coregistration in the presence of a rotation and a translation between them. To this aim, let us indicate with $z=x+j y$ the complex variable representing the Cartesian coordinates $x$ and $y$. By doing so the generic image can be directly indicated as $\boldsymbol{I}(z)$, with $z \in \mathbb{C}$. As a consequence, taking into consideration two images representing the same scene, $\boldsymbol{I}_{m} \in \mathbb{C}^{K \times N}$ (master) and $\boldsymbol{I}_{s} \in \mathbb{C}^{K \times N}$ (slave), we can model the effect of pixels translation and rotation from one image with respect to the other, due to the different sensor trajectory experienced during the acquisition process, as

$$
\boldsymbol{I}_{s}(z)=\boldsymbol{I}_{m}((z-\delta) / \alpha)+\boldsymbol{E}(z),
$$

where $\boldsymbol{E}(z)$ is the error image that is representative of noise and the different scattering properties of the considered pair, with the master and slave images labeled with the subscripts $m$ and $s$, respectively. Moreover, the complex parameter $\delta=\delta_{x}+j \delta_{y}$ is the complex displacement accounting respectively for horizontal $\delta_{x}$ and vertical $\delta_{y}$ displacements, whereas $\alpha=\gamma \exp [j \theta]$ is a complex scalar factor accounting for rotation, described by the rotation angle $\theta$ and the zooming factor $\gamma$. Let us note that, since SAR images are not affected by zooming effect the modulus $\gamma$ should be equal to 1 . Now, the problem to be solved consists in estimating the unknown complex parameters $\delta$ and $\alpha$. To do this, we divide the two quoted images into $L$ (with $L \geq 2$ ) rectangular blocks (or patches) of size $W_{1} \times W_{2}$, say $\boldsymbol{P}_{m, l} \in \mathbb{C}^{W_{1} \times W_{2}}$ and $\boldsymbol{P}_{s, l} \in \mathbb{C}^{W_{1} \times W_{2}}, l=1, \ldots, L$, respectively. For small blocks, the spatial deformation due to a slight rotation can approximate a pure translation, basically depending on the block center's position that shifts in the whole image [12].

Then, to have a coarse estimation of the 2D displacement of each block, the 2D spatial cross-correlation, $C_{l} \in$ $\mathbb{C}^{\left(2 W_{1}-1\right) \times\left(2 W_{2}-1\right)}$, between each patch of the master and the homologous of the slave is computed [13]. Precisely, the position of the maximum values in their modulus, say $\left(h_{0, l}, p_{0, l}\right) l=1, \ldots, L$, are individuated and selected to form the overall displacement field in $\zeta_{l}=h_{0, l}+j p_{0, l}, l=1, \ldots, L$. By doing so, the overall displacement field between the two images is computed solving an over-determined linear system of $L$ equations in 4 unknowns, i.e.,

$$
\alpha z_{l}+\delta=\zeta_{l}, \quad l=1, \ldots, L,
$$

where $z_{l}=x_{l, c}+j y_{l, c}$ is the complex number representing the Cartesian coordinates, $\left(x_{l, c}, y_{l, c}\right)$ of the center of the $l$-th block in the master image. The above system of equations can be also cast in a more compact matrix form, i.e.,

$$
Z p=\zeta
$$

with

$$
\boldsymbol{Z}=\left[\begin{array}{cc}
z_{1} & 1 \\
\vdots & \vdots \\
z_{L} & 1
\end{array}\right]
$$

$\boldsymbol{p}=[\alpha, \delta]^{T}$, and $\boldsymbol{\zeta}=\left[\zeta_{1}, \ldots, \zeta_{L}\right]^{T}$ (with the coordinates $\zeta_{1}, \ldots, \zeta_{L}$ expressed with respect to the reference system centered at the image center). It is also worth recalling that the coarse estimate can be refined with a fine estimate of the 2D paraboloid vertex obtainable in closed-form from the six neighbors including $\left(h_{0, l}, p_{0, l}\right)$ [14].

It is now important to underline that (3) assumes that all the extracted patches impact equally on the designed system. However, due to noise or decorrelation effects, the contributions of the different patches could be differently weighted. In order to account for these effects we introduce a weighting in (3) obtaining

$$
\operatorname{diag}(w) Z p=\operatorname{diag}(w) \zeta,
$$

where $\boldsymbol{w}=\left[w_{1}, \ldots, w_{L}\right]$ is the vector containing the weights applied to each extracted patch. The weights could be selected based on coherence magnitude or on prior geospatial knowledge in order to reduce the impact of corrupted or less reliable patches to the overall system. Note that, for $\boldsymbol{w}=[1, \ldots, 1]^{T}$, (4) reduces to (3). In addition, since SAR images are not affected by zooming effect (in fact, the quoted images pair has the same resolution and the same swath on terrain), we also enforce the constraint $\gamma=1$ to (4), that becomes a CLS problem, namely

$$
\left\{\begin{array}{l}
\arg \min _{\boldsymbol{p}}\|\boldsymbol{A} \boldsymbol{p}-\operatorname{diag}(\boldsymbol{w}) \boldsymbol{\zeta}\|^{2} \\
\text { s.t. } \boldsymbol{p}^{\dagger} \boldsymbol{D} \boldsymbol{p}-1=0
\end{array},\right.
$$

with

$$
\boldsymbol{D}=\left[\begin{array}{ll}
1 & 0 \\
0 & 0
\end{array}\right] \quad \text { and } \quad \boldsymbol{A}=\operatorname{diag}(\boldsymbol{w}) \boldsymbol{Z}
$$

The general formulation of the CLS problem in (5) together with the derivation of its closed-form solution provided in the following represents the main technical new contribution of this letter.

From [10, Theorem 3.2], $\boldsymbol{p} \in \mathbb{C}^{2}$ is an optimal solution to 
CLS in (5) if and only if there exists $\beta \in \mathbb{R}$ such that

$$
\left\{\begin{array}{l}
\left(\boldsymbol{A}^{\dagger} \boldsymbol{A}+\beta \boldsymbol{D}\right) \boldsymbol{p}=\boldsymbol{A}^{\dagger} \operatorname{diag}(\boldsymbol{w}) \boldsymbol{\zeta} \\
\boldsymbol{p}^{\dagger} \boldsymbol{D} \boldsymbol{p}-1=0 \\
\boldsymbol{A}^{\dagger} \boldsymbol{A}+\beta \boldsymbol{D} \succeq \mathbf{0}
\end{array} .\right.
$$

We also assume that $\boldsymbol{A}$ is full column rank, i.e., $\boldsymbol{A}^{\dagger} \boldsymbol{A}$ is full rank. It follows that the optimal solution to CLS is

$$
\boldsymbol{p}^{\star}=\left(\boldsymbol{A}^{\dagger} \boldsymbol{A}+\beta \boldsymbol{D}\right)^{-1} \boldsymbol{A}^{\dagger} \operatorname{diag}(\boldsymbol{w}) \boldsymbol{\zeta}
$$

with $\beta$ solution of $\varphi(\beta)=0, \beta \in \mathcal{J} \subseteq \mathbb{R}$, and $\varphi(\beta)=\boldsymbol{p}^{\dagger} \boldsymbol{D} \boldsymbol{p}$ 1. The interval J consists of all $\beta$ for which $\boldsymbol{A}^{\dagger} \boldsymbol{A}+\beta \boldsymbol{D}$ is positive definite, which implies that

$$
\beta \in\left(-\frac{1}{\lambda_{1}\left(\boldsymbol{D}, \boldsymbol{A}^{\dagger} \boldsymbol{A}\right)},+\infty\right) .
$$

The roots of the equation $\varphi(\beta)=0$ can be found in closedform (see Appendix A for the derivations), then the global optimal solution is chosen as the best of the two possible solutions. More precisely, if both the two solutions of $\varphi(\beta)=$ 0 are feasible, say $\beta_{1}, \beta_{2}$, the optimal solution is chosen as the one that minimize the Least Squares (LS) problem, i.e.,

$$
i^{\star}=\arg \min _{i=1,2}\left\|\boldsymbol{A} \boldsymbol{p}^{\star}\left(\beta_{i}\right)-\operatorname{diag}(\boldsymbol{w}) \boldsymbol{\zeta}\right\|^{2},
$$

and hence

$$
\boldsymbol{p}^{\star}=\left(\boldsymbol{A}^{\dagger} \boldsymbol{A}+\beta_{i^{\star}} \boldsymbol{D}\right)^{-1} \boldsymbol{A}^{\dagger} \operatorname{diag}(\boldsymbol{w}) \boldsymbol{\zeta} .
$$

Before concluding this section, the proposed coregistration algorithm for rotated SAR images is pictorially shown in Fig. 1, whereas Algorithm 1 summarizes its main steps. Moreover, some considerations on the computational complexity are of interest. First of all, it is worth highlighting that the operations of finding the roots $\beta_{1}$ and $\beta_{2}$ of the equation $\varphi(\beta)=0$ satisfying (8), computing $\boldsymbol{p}^{\star}\left(\beta_{i}\right), i=1,2$, and determining $i^{\star}$ can be performed in closed-form $(2 \times 2$ matrix inversion and evaluation of the objective function for at most two points). Furthermore, the cross-correlation between the couple of patches can be also efficiently obtained [6].

\section{Performance Assessment}

This section is devoted to the evaluation of the effectiveness of the proposed coregistration algorithm for rotated and translated images. Precisely, the tests are conducted on the Gotcha Volumetric SAR Data Set V1.0 [11], acquired with a carrier frequency of $9.6 \mathrm{GHz}$ and $640 \mathrm{MHz}$ bandwidth, full azimuth coverage, eight different elevation angles and full polarization. The observed scene comprises several civilian vehicles together with calibration targets. For the analyses herein conducted the aperture has been divided in sub apertures of $4^{\circ}$ in azimuth, leading to approximately equal range-azimuth resolution cells of $23 \mathrm{~cm}$. The image of size $501 \times 501$ pixels is available in the four polarizations $(\mathrm{HH}, \mathrm{VV}, \mathrm{HV}, \mathrm{VH})$, but, without loss of generality, we focus our attention on the $\mathrm{HH}$ selecting also the first $4^{\circ}$ aperture image at the first and second elevation passes. The numerical tests conducted in this letter utilize the first pass image as master, whereas the slave is $\overline{\text { Algorithm } 1 \text { Coregistration Algorithm of Rotated and Trans- }}$ lated SAR Images

Input: Master SAR image $\boldsymbol{I}_{m} \in \mathbb{C}^{K \times N}$, and slave SAR image $\boldsymbol{I}_{s} \in \mathbb{C}^{K \times N}$;

Output: Coregistered slave SAR image $\boldsymbol{I}_{c} \in \mathbb{C}^{K \times N}$;

1: Extraction of $L$ patches from the images $\boldsymbol{I}_{m}$ and $\boldsymbol{I}_{s}$;

2: Computation of the 2D cross-correlation $C_{l}$ between each couple of patches;

3: Identification of the 2D cross-correlation's peak for each patch, $\left(h_{0, l}, p_{0, l}\right), l=1, \ldots, L$;

4: Find the roots $\beta_{1}$ and $\beta_{2}$ of the equation $\varphi(\beta)=0$ satisfying (8);

5: Compute $\boldsymbol{p}^{\star}\left(\beta_{i}\right), i=1,2$;

6: Determine $i^{\star}$ through (9) and select the optimal solution $\boldsymbol{p}^{\star}\left(\beta_{i^{\star}}\right)$;

7: Estimation of parameters $\theta, \delta_{x}$, and $\delta_{y}$, say $\hat{\theta}, \hat{\delta}_{x}$, and $\hat{\delta}_{y}$;

8: Translation and rotation of the slave image $\boldsymbol{I}_{s}$ by a quantity $\left(\hat{\delta}_{x}, \hat{\delta}_{y}, \hat{\theta}\right)$ to obtain $\boldsymbol{I}_{c}$.

generated from that at second pass by means of a counterclockwise rotation by an angle $\theta$. Moreover, since the rotation produces a non-integer translation of the pixels, the resulting image is also re-sampled with a nearest neighbor interpolation. This effect can be appreciated through the observation of Fig. 2 which shows a portion of the considered master and slave images, where the impact of a respective rotation of $2^{\circ}$ is clear.

Therefore, the effectiveness of the proposed method is assessed utilizing as figure of merit the coherence magnitude between the registered couple of images [15] given by

$$
\rho=\left|\frac{\sum_{k=0}^{K-1} \sum_{n=0}^{N-1} \boldsymbol{I}_{m}(k, n) \boldsymbol{I}_{c}^{*}(k, n)}{\sqrt{\sum_{k=0}^{K-1} \sum_{n=0}^{N-1}\left|\boldsymbol{I}_{m}(k, n)\right|^{2}} \sqrt{\sum_{k=0}^{K-1} \sum_{n=0}^{N-1}\left|\boldsymbol{I}_{c}(k, n)\right|^{2}}}\right| .
$$

Accordingly, Fig. 3 reports the coherence magnitude $\rho$ of the registered couples as a function of the rotation angle varying in the interval $\left[0^{\circ}, 2^{\circ}\right]$, small enough to approximate block motion as a pure shift. The curves compare the proposed algorithm with all equal weights (i.e., $\boldsymbol{w}=[1, \ldots, 1]^{T}$ ), labeled with CLS, and the algorithm that performs the translation only indicated by the Cross-Correlation Peak (CCP). Moreover, three different sizes for the extracted patches are considered, viz. $W=W_{1}=W_{2}=(22,44,66)$. Interestingly, the curves show that the proposed algorithm is able to give good results in terms of re-alignment of the images under test, with high coherence values close to the benchmark given by the coherence before the induced rotation, and a huge gain over that computed from the rotated not-registered couple (indicated as not-registered in the figure). In addition, the proposed method is able to provide better results than those given by the classic CCP approach. Finally, higher patch size provides a more robust behavior with respect to the rotation angle. This can be justified observing that the conventional multiply-and-add estimator underestimates the 


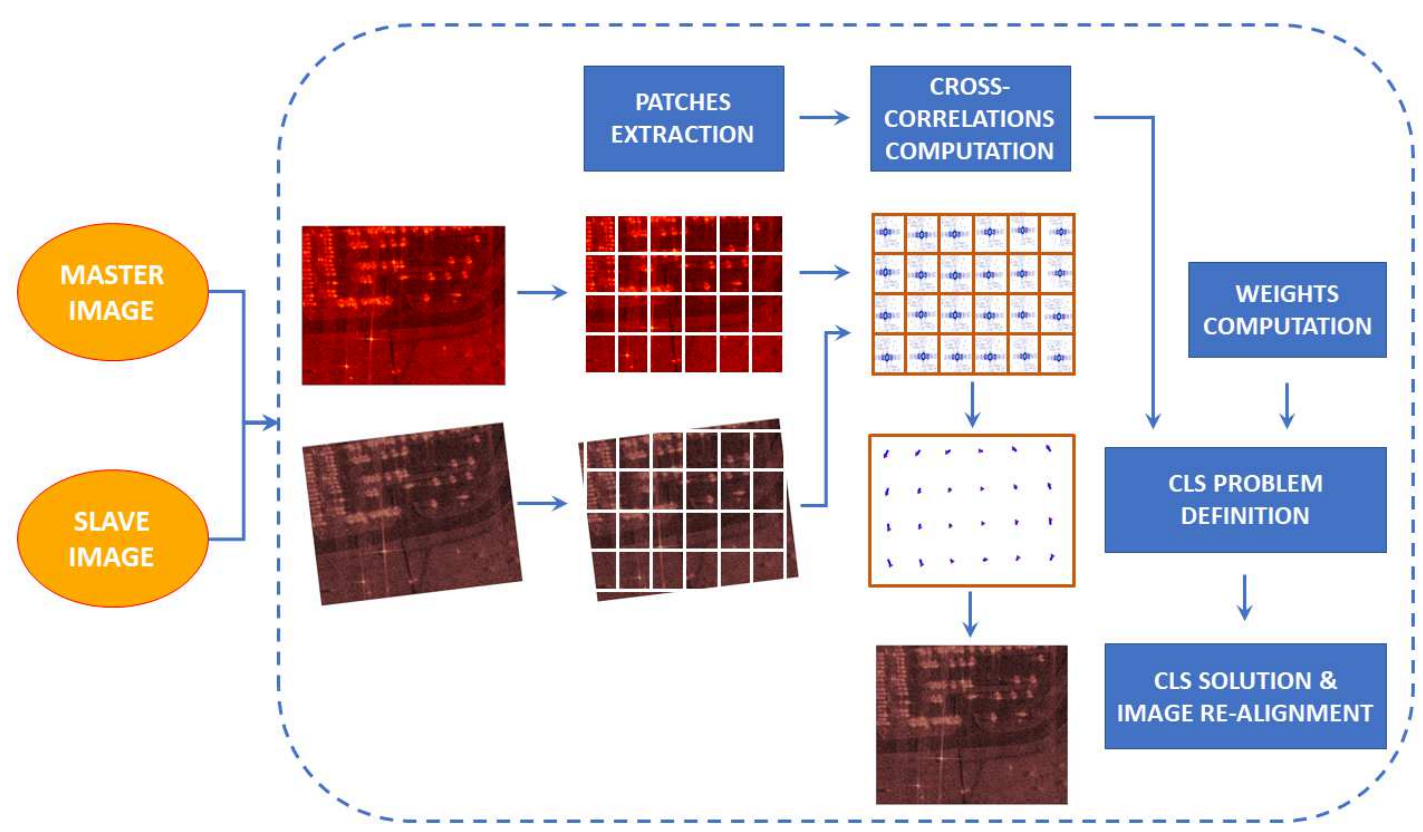

Figure 1. Block scheme of proposed SAR images coregistration algorithm in the presence of rotation and translation effects.
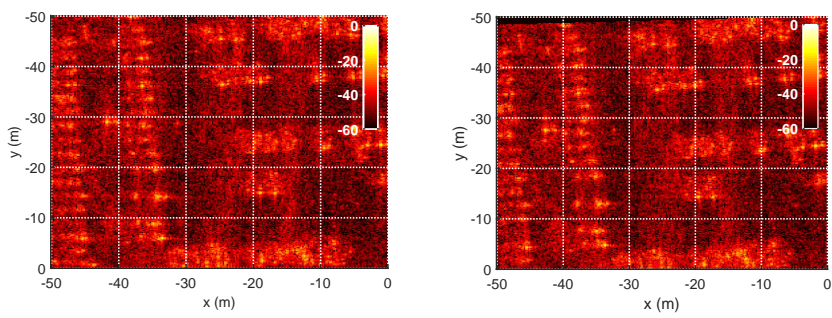

Figure 2. Portion of master and slave images (modulus expressed in $\mathrm{dB}$ ). Slave image obtained through a counterclockwise rotation of $2^{\circ}$

cross-correlation tails because of the triangular windowing effect. Moreover, the estimated shifts of the external blocks affect the rotation factor estimate much more than central blocks. At the image corners of a $M \times M$ image, the motion vectors move of $s_{c}=(\sqrt{2} / 2) M \sin (\theta)$. Therefore, if the patch size is $L=2 s_{c}$, the estimated correlation peak will be half of the true peak, possibly resulting in its ambiguous detection for some patches. Conversely, if the patch size increases such effect becomes more and more negligible, as observed in Fig. 3.

To further emphasize the benefits of the proposed methodology, Table I synthetically gives the estimates of the parameters $\theta, \delta_{x}$, and $\delta_{y}$, for the CLS and CCP algorithms for a simulation setting composed by $\theta=\left(1^{\circ}, 2^{\circ}\right), \delta_{x}=0$, and $\delta_{y}=0$. From the estimated values, the superiority of the CLS algorithm over the CCP, which completely fails in this situation, is quite evident.

Last analysis herein presented is the impact of noise on the proposed algorithm in terms of Mean Square Error (MSE) of the estimated vector $\boldsymbol{p}$. Precisely, the considered couple of images is composed by the same master as before and with the slave obtained counterclockwise rotating the master and with the addition of complex white Gaussian noise. This

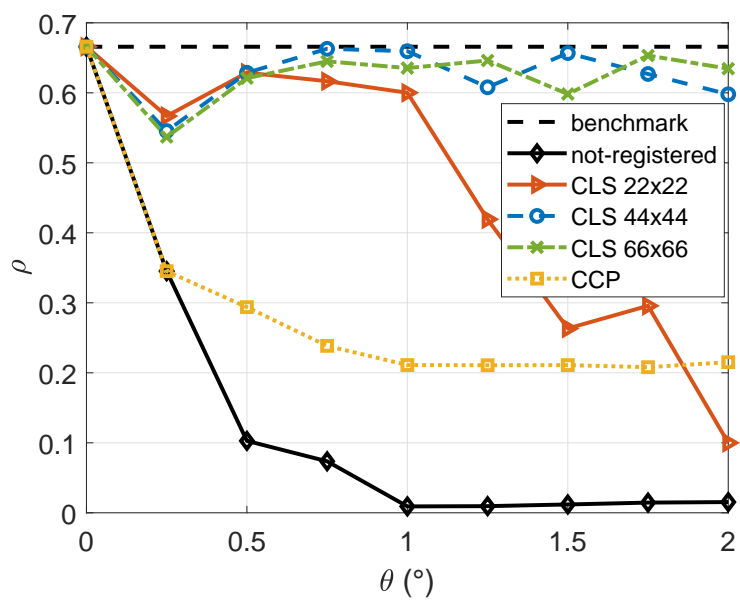

Figure 3. Coherence magnitude versus rotation angle.

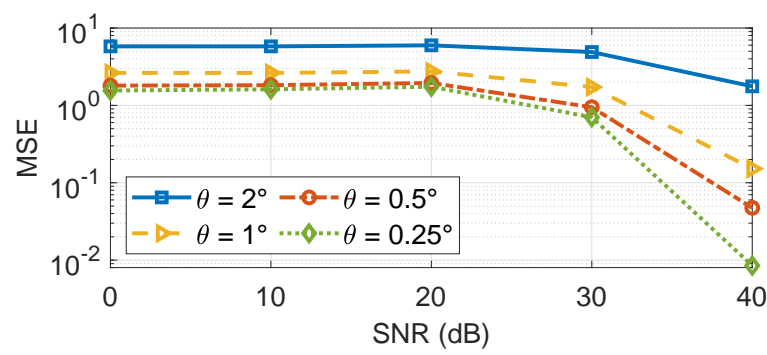

Figure 4. MSE vs SNR of the estimated $\boldsymbol{p}$ for several rotation angles.

analysis is given in Fig. 4 that plots MSE versus Signal to Noise Ratio (SNR) for several rotation angles. The curves clearly emphasize the negative impact of noise on registration performance, with MSE increasing with decreasing SNR level. 
Table I

ESTIMATED COREGISTRATION PARAMETERS.

\begin{tabular}{ccccc}
\hline & & $\hat{\theta}\left({ }^{\circ}\right)$ & $\hat{\delta}_{x}$ & $\hat{\delta}_{y}$ \\
\hline a) & true & 1 & 0 & 0 \\
\cline { 2 - 5 } & CLS $(W=22)$ & 0.933 & -0.118 & -0.034 \\
\cline { 2 - 5 } & CLS $(W=44)$ & 0.996 & 0.026 & -0.040 \\
\cline { 2 - 5 } & CLS $(W=66)$ & 0.965 & -0.016 & 0.023 \\
\hline & CCP & - & -2 & -1 \\
\hline & true & 2 & 0 & 0 \\
\cline { 2 - 5 } & CLS $(W=22)$ & 1.536 & -0.496 & -0.414 \\
\cline { 2 - 5 } & CLS $(W=44)$ & 1.927 & -0.098 & 0.100 \\
\hline & 1.974 & -0.080 & 0.119 \\
\hline
\end{tabular}

\section{CONClusions}

This letter has described a new method to coregister SAR images affected by shift and rotation effects. The procedure starts dividing the two images into several patches; then, the displacement vector for each pair is evaluated and a constrained LS problem is derived, forcing the absence of zooming effects. The corresponding solution has been efficiently found thanks to the theory of GTRS. The performance of the proposed procedure have been assessed on real-recorded SAR data evaluating the coherence between the images after the coregistration process. Interesting results have been obtained also in comparison with the classic approach based on the rigid translation in range and azimuth direction of the slave image. The impact of noise has also been analyzed in terms of MSE of the estimated parameters vector. Possible future researches might involve the tests on different SAR images (e.g., in lower bands and with different observed scenarios), as well as the investigation of non-equal weights and the optimum patch size.

\section{AKNOWLEDGMENT}

The authors would like to thank the Editor and Reviewers for the interesting comments that have helped to improve this letter.

\section{APPENDIX}

\section{A. Procedure to find the roots of $\varphi(\beta)=0$}

Let consider the eigenvalue decomposition of the matrix $\boldsymbol{A}^{\dagger} \boldsymbol{A}, \boldsymbol{A}^{\dagger} \boldsymbol{A}=\boldsymbol{U} \boldsymbol{\Lambda} \boldsymbol{U}^{\dagger}$. The solution $\boldsymbol{p}$ can be written as

$$
\begin{aligned}
\boldsymbol{p} & =\left(\boldsymbol{U} \boldsymbol{\Lambda} \boldsymbol{U}^{\dagger}+\beta \boldsymbol{D}\right)^{-1} \boldsymbol{A}^{\dagger} \operatorname{diag}(\boldsymbol{w}) \boldsymbol{\zeta} \\
& =\boldsymbol{U}(\boldsymbol{\Lambda}+\beta \boldsymbol{D})^{-1} \boldsymbol{U}^{\dagger} \boldsymbol{A}^{\dagger} \boldsymbol{d i a g}(\boldsymbol{w}) \boldsymbol{\zeta} \\
& =\left(\begin{array}{cc}
u_{11} & u_{12} \\
u_{21} & u_{22}
\end{array}\right)\left(\begin{array}{cc}
\frac{1}{\lambda_{1}+\beta} & 0 \\
0 & \frac{1}{\lambda_{2}}
\end{array}\right) \underbrace{\boldsymbol{U}^{\dagger} \boldsymbol{A}^{\dagger} \boldsymbol{d i a g}(\boldsymbol{w}) \boldsymbol{\zeta}}_{\boldsymbol{h}} \\
& =\left(\begin{array}{l}
\frac{u_{11} h_{1}}{\lambda_{1}+\beta}+\frac{u_{12} h_{2}}{\lambda_{2}} \\
\frac{u_{21} h_{1}}{\lambda_{1}+\beta}+\frac{u_{22} h_{2}}{\lambda_{2}}
\end{array}\right) .
\end{aligned}
$$

Now, we have to solve the problem $\boldsymbol{p}^{\dagger} \boldsymbol{D} \boldsymbol{p}-1=0$, namely

$$
\begin{aligned}
& \left(\frac{u_{11}^{*} h_{1}^{*}}{\lambda_{1}+\beta}+\frac{u_{12}^{*} h_{2}^{*}}{\lambda_{2}}, \frac{u_{21}^{*} h_{1}^{*}}{\lambda_{1}+\beta}+\frac{u_{22}^{*} h_{2}^{*}}{\lambda_{2}}\right)\left(\begin{array}{ll}
1 & 0 \\
0 & 0
\end{array}\right)\left(\begin{array}{l}
\frac{u_{11} h_{1}}{\lambda_{1}+\beta}+\frac{u_{12} h_{2}}{\lambda_{2}} \\
\frac{u_{21} h_{1}}{\lambda_{1}+\beta}+\frac{u_{22} h_{2}}{\lambda_{2}}
\end{array}\right) \\
& -1=0,
\end{aligned}
$$

that becomes

$$
\left(\frac{u_{11}^{*} h_{1}^{*}}{\lambda_{1}+\beta}+\frac{u_{12}^{*} h_{2}^{*}}{\lambda_{2}}\right)\left(\frac{u_{11} h_{1}}{\lambda_{1}+\beta}+\frac{u_{12} h_{2}}{\lambda_{2}}\right)-1=0,
$$

whose solution is easy to obtain.

\section{REFERENCES}

[1] A. Moreira, P. Prats-Iraola, M. Younis, G. Krieger, I. Hajnsek, and K. P. Papathanassiou, "A Tutorial on Synthetic Aperture Radar," IEEE Geoscience and Remote Sensing Magazine, vol. 1, no. 1, pp. 6-43, 2013.

[2] Y. Wang, Q. Yu, and W. Yu, "An Improved Normalized Cross Correlation Algorithm for SAR Image Registration," in 2012 IEEE International Geoscience and Remote Sensing Symposium (IGARSS). IEEE, 2012, pp. 2086-2089.

[3] Z. Zhang, H. Liu, L. Zhang, S. Wang, Z. Li, and J. Wu, "A Large Width SAR Image Registration Method Based on the Complex Correlation Function," in 2016 IEEE International Geoscience and Remote Sensing Symposium (IGARSS). IEEE, 2016, pp. 6476-6479.

[4] R. Scheiber and A. Moreira, "Coregistration of Interferometric SAR Images using Spectral Diversity," IEEE Transactions on Geoscience and Remote Sensing, vol. 38, no. 5, pp. 2179-2191, September 2000.

[5] D. Li and Y. Zhang, "A Fast Offset Estimation Approach for InSAR Image Subpixel Registration," IEEE Geoscience and Remote Sensing Letters, vol. 9, no. 2, pp. 267-271, 2011.

[6] E. H. Peterson, G. Fotopoulos, A. Schmitt, R. E. Zee, and A. Roth, "Registration of Multi-Frequency SAR Imagery using Phase Correlation Methods," in 2011 IEEE International Geoscience and Remote Sensing Symposium. IEEE, 2011, pp. 3708-3711.

[7] E. Sansosti, P. Berardino, M. Manunta, F. Serafino, and G. Fornaro, "Geometrical SAR Image Registration," IEEE Transactions on Geoscience and Remote Sensing, vol. 44, no. 10, pp. 2861-2870, October 2006.

[8] H. Goncalves, L. Corte-Real, and J. A. Goncalves, "Automatic Image Registration Through Image Segmentation and SIFT," IEEE Transactions on Geoscience and Remote Sensing, vol. 49, no. 7, pp. 2589-2600, July 2011.

[9] S. Paul and U. C. Pati, "A Block-Based Multifeature Extraction Scheme for SAR Image Registration," IEEE Geoscience and Remote Sensing Letters, vol. 15, no. 9, pp. 1387-1391, September 2018.

[10] More' J. J., "Generalizations of the Trust Region Subproblem," Optim. Methods Softw., vol. 2, pp. 189-209, August 1993.

[11] E. Ertin, C. D. Austin, S. Sharma, R. L. Moses, and L. C. Potter, "GOTCHA Experience Report: Three-Dimensional SAR Imaging with Complete Circular Apertures," in Algorithms for Synthetic Aperture Radar Imagery XIV. International Society for Optics and Photonics, 2007, vol. 6568, p. 656802 .

[12] G. Giunta and U. Mascia, "Estimation of Global Motion Parameters by Complex Linear Regression," IEEE Transactions on Image Processing, vol. 8, no. 11, pp. 1652-1657, 1999.

[13] R. C. Gonzalez and R. E. Woods, Digital Image Processing, Pearson/Prentice Hall, Upper Saddle River, NJ, 3rd edition, 2008.

[14] L. Pallotta, G. Giunta, and C. Clemente, "Subpixel SAR Image Registration Through Parabolic Interpolation of the 2-D Cross Correlation," IEEE Transactions on Geoscience and Remote Sensing, 2020.

[15] R. Touzi, A. Lopes, J. Bruniquel, and P. W. Vachon, "Coherence Estimation for SAR Imagery," IEEE Transactions on Geoscience and Remote Sensing, vol. 37, no. 1, pp. 135-149, January 1999. 